\title{
SAR, OPTICAL AND LIDAR DATA FUSION FOR THE HIGH RESOLUTION MAPPING OF NATURAL PROTECTED AREAS
}

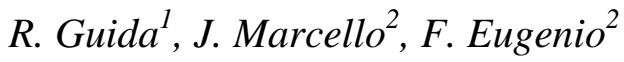 \\ ${ }^{1}$ Surrey Space Centre, University of Surrey, Guildford, United Kingdom \\ r.guida@surrey.ac.uk \\ ${ }^{2}$ Instituto de Oceanografía y Cambio Global (IOCAG). Universidad de Las Palmas de Gran Canaria, \\ Spain
}

\begin{abstract}
The singular characteristics of the Canarian archipelago (Spain) have allowed the development of a unique biological richness. Almost half of its territory is protected to preserve the natural environment. In this paper, different approaches to consider fusion of multi-sensor data are considered and corresponding methodologies described. The application to real datasets over Canarian islands is undergoing and fusion maps will be presented at the conference while preliminary classification results with multispectral data are described here.
\end{abstract}

Index Terms - Data Fusion, TerraSAR-X, Worldview2, LIDAR, SVM.

\section{INTRODUCTION}

The accurate mapping of vegetation covers in semi-arid natural protected areas is a challenging topic as, typically, such environments are sparsely vegetated and mainly composed by small shrubs of different species growing together in favorable places. In addition, the low leaf area limits the applicability of the VNIR vegetation indices. Therefore, it is difficult to retrieve quantitative information on vegetation type, cover and biomass due to the mixing contributions in the resolution cell and the dominance of soil background reflectance.

The launch of different very high resolution remote sensing missions has enabled the acquisition of images with spatial resolutions in the order of the meter or below, initiating a completely new way to approach traditional applications, such as mapping, urban planning, environment, agriculture, geology, resource management, prediction risks, protection of natural spaces, etc.

This paper describes an attempt to use high resolution optical and SAR imagery together to assist in mapping vegetation covers in protected areas. Classification techniques based on optical satellite sensors have some limitations in distinguishing between vegetation types being based mainly on the spectral information of the VNIR region in which spectral signatures of different species may present high similarity. Hence, radar imagery, with its ability to extract textural information and distinguish the different scattering mechanisms of the target object, can support discrimination of vegetation types on the base of their geometric properties. In addition, classification can be further improved with additional datasets, if available. For example, canopy heights, biomass measurements, and leaf area can all be studied using airborne LIDAR systems. In this project, such data is also incorporated to discriminate between different shrubs or tree species using the height information.

\section{STUDY AREA}

Two protected areas have been selected for this study (Figure 1): the Teide National Park (Tenerife Island) and the Maspalomas Special Natural Reserve (Gran Canaria Island). The Teide National Park was created in 1954 in order to protect this spectacular landscape of great ecological value which lies at the foot of the colossal volcano $(3718 \mathrm{~m}$ height).

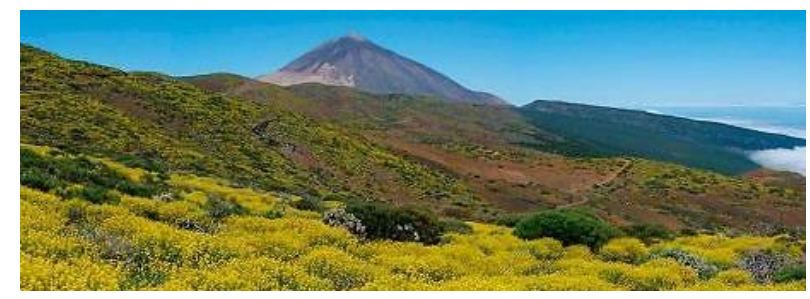

(a)

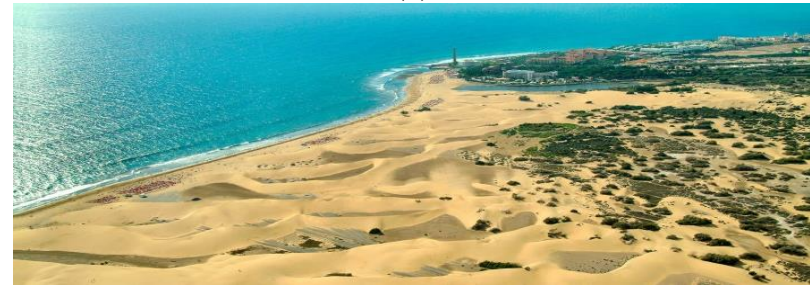

(b)

Figure 1. Natural protected areas: (a) Teide National Park and (b) Maspalomas Special Natural Reserve. 
Plant and animal species are unique and, in particular, the endemic plants are adapted to the tough environmental conditions on the volcano such as high altitude, intense sunlight and extreme temperature variations.

The Maspalomas Special Natural Reserve embraces 403.9 hectares of sand dunes, a lagoon of great ecological value and a bird nesting centre. This is a well preserved area but strongly influenced by human presence, which represents a serious threat to its survival. A large part of the flora is protected by national and regional regulations.

\section{MAPPING METHODOLOGY}

\subsection{Data}

The VNIR data selected for the analysis was coming from the WorldView-2 (WV-2) satellite acquired on 16 May 2011. The sensor has a spatial resolution of $0.46 \mathrm{~m}$ at nadir $\left(0.56 \mathrm{~m}\right.$ at $20^{\circ}$ off-nadir) for the panchromatic band, while the multispectral (MS) imagery is captured with a resolution of $1.8 \mathrm{~m}$ at nadir (2.4 m off-nadir). The 8 spectral bands include the 4 traditional visible to near infrared bands, and additional four spectral bands: Coastal Blue $(400-450 \mathrm{~nm})$, Yellow (585-625 nm), Red Edge (705-745 nm) and another NIR2 (860-1040 nm) band.

As regards SAR data, two options were available to authors: TerraSAR-X (TSX) data in X-band (around 9 $\mathrm{GHz}$ ), acquired in either Spotlight (around $1 \mathrm{~m} \times 1 \mathrm{~m}$ resolution) or Stripmap mode (around $3 \mathrm{~m} \times 3 \mathrm{~m}$ resolution) in the years 2007/2008 and Sentinel-1 data in C-band (around $5 \mathrm{GHz}$ ) acquired in Stripmap (5m x 5m resolution) and Interferometric Wide Swath or Enhanced Wide Swath modes (presenting, respectively, $5 \mathrm{~m} \times 20 \mathrm{~m}$ and $20 \mathrm{~m}$ x $40 \mathrm{~m}$ resolution) in November and December 2014. After an initial attempt to work on Sentinel data in order to minimize the temporal gap between radar and multispectral datasets, the authors redirected their attention on TSX Spotlight data as spatial resolution in the order of meter looked more important in this initial test given the kind of classes that had to be identified. A couple of TSX spotlight acquisitions were considered good for the case study in terms of overlapping areas and an example is shown in Figure 2 where (a) displays the TSX image acquired on 10 May 2008 and (b) shows how it overlaps with the WV-2. The season is preserved but the temporal gap of 3 years will have to be considered in the discussion of fusion results at the conference.

LIDAR flight campaign over Canary Islands was carried out during 2012 with an airborne sensor Leica ALS60 and data are provided by GRAFCAN. The average density is of 1.20 points per square meter and $0.8 \mathrm{ppsm}$ at the nadir. The mean accuracies of registered points range around 0.60 meters in planimetry and 0.20 meters in altimetry. Figure 3 shows WV-2 and LiDAR data used for mapping a specific area of the Teide Park. (a)

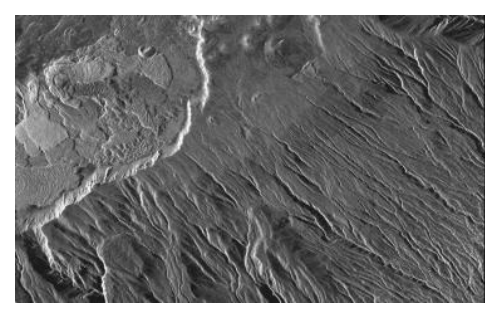

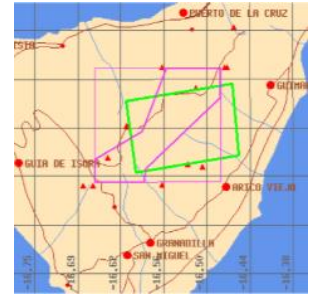

(b)
Figure 2 - (a) preview of the TSX dataset over Teide (10 May 2008; (b) overlapping between the two datasets considered, TSX (green frame) and WV-2 (scarlet polygon).

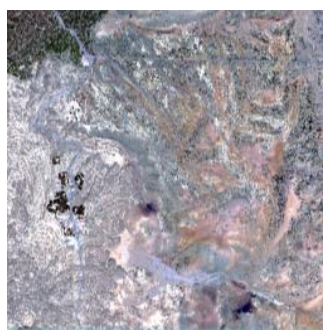

(a)

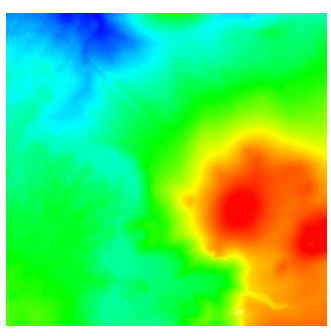

(b)
Figure 3. Teide Park: (a) Worldview-2 color composite (16 May 2011), (b) LIDAR maximum height (2012).

In addition, a field campaign was conducted during 2015 at each protected area to retrieve the maximum spectral and spatial information.

\subsection{Methodology}

As indicated, the objective has been to develop and implement a multimode image processing methodology for the monitoring of natural protected areas using high resolution VNIR, SAR and LIDAR data. Different approaches have been considered in which the fusion is performed at different levels and with different and gradual inclusion of the datasets available. In the conceptually simplest method, the fusion of the datasets is performed at thematic map level, meaning that a classification map is derived by each dataset independently and, only later, the thematic maps are merged.

Alternatively, two other more complex procedures are also currently under development. The first regards the fusion of SAR, Multispectral and Panchromatic datasets through application of the generalized IHS (Intensity, Hue, Saturation) [1] transform to the Multispectral bunch. This step produces a generalized intensity that is later properly sharpened by the Panchromatic channel (through wavelet transform) and modulated by the texture of the SAR dataset. In the second procedure, raster masks are produced using 
LiDAR information about intensity, Digital Elevation Model, and multiple returns from the scenario. Each mask is then used in conjunction with either the SAR dataset or the Multispectral one in a Support Vector Machine (SVM) classifier and the obtained classification maps are finally properly combined.

All datasets have undergone proper pre-processing. For the first approach, Worldview-2 bands have been preprocessed to eliminate radiometric, atmospheric and geometric disturbances to the signal. In particular, the $6 \mathrm{~S}$ algorithm [2] has been applied to eliminate the absorption, scattering and refraction effects of the atmosphere in the different spectral bands. A topographic correction algorithm [3] has been used to remove the effects of the relief. Finally, in order to increase the spatial resolution with minimum spectral distortion, pan-sharpening techniques $[4,5]$ have been considered. In particular de Gram-Schmidt (GS) algorithm was employed. Classification of WV-2 data alone for the first approach was performed applying 4 supervised methods [6]: Mahalanobis distance (MhD), Maximum Likelihood (ML), Spectral Angel Mapper (SAM) and Support Vector Machines (SVM). SVM have proven to be very effective in solving complex classification problems, mainly due to the fact that this technique does not require an estimation of the statistical distribution of classes and the ability to handle limited amount or quality of training samples [7, 8].

SAR datasets have been radiometrically calibrated, multilooked and ground-projected. Terrain correction has been also applied.

Coregistration and resizing of the different datasets have also been performed to allow implementation of either fusion procedure.

\section{RESULTS}

As anticipated, the implementation of the fusion approaches is not complete at the time of writing, so final results will be presented at the conference while intermediate classification maps obtained from WV-2 after a comparative analysis are here presented for the Teide Park.

Four supervised classifiers were applied to the 6 combinations of original MS and GS pansharpened bands for each area of study. In the analysis of the Teide Park, the following thematic classes were considered: pine (dark green), broom (light green), pajonera (yellow), rosalillo (violet), bare soil (orange), built soil (red) and asphalt (black). Figure 4 displays the vegetation types considered of interest to the park. It is important to highlight the complexity to discriminate them given their small size, spectral similarity and mixture of contributions at each pixel. Visual inspection shows that SVM classification provides the best quality maps. A quantitative assessment was also conducted and the confusion matrix and the kappa coefficient were computed.

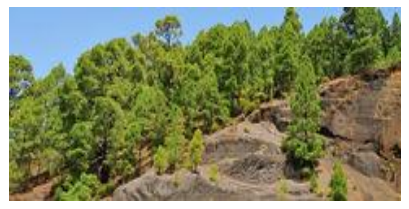

(a)

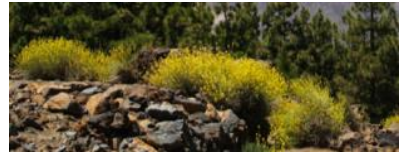

(c)

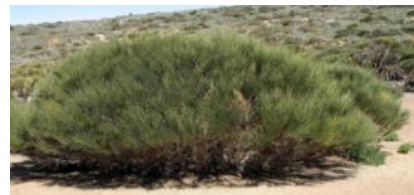

(b)

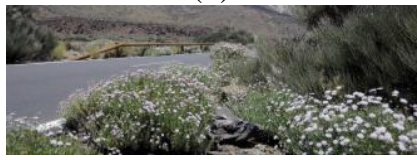

(d)
Figure 4. Vegetation classes: (a) pine, (b) broom, (c) pajonera and (d) rosalillo.

The overall accuracy values are presented in Table I. An example of each classifier, using the optimal combination of input bands, is displayed in Figure 5. The inclusion of additional information (texture or indices) does not always involve an improvement on the thematic maps obtained. In general, SVM provides better results and pixel-level fusion clearly improves the visual quality (Figure 6) but the accuracy improvement is not as clear, however it depends on the test regions selected.

Table I. Overall accuracy of different classifiers applied to combinations of the original and fused WV-2 data.

\begin{tabular}{|l|c|c|c|c|}
\hline \multicolumn{1}{|c|}{ Bands: } & MhD & ML & SAM & SVM \\
\hline MS bands & 86,2 & 90.9 & $\mathbf{8 4 . 6}$ & 91.1 \\
MS+Texture & 85.4 & $\mathbf{9 1 . 6}$ & $\mathbf{8 4 . 6}$ & 89.3 \\
MS+Indices & $\mathbf{8 6 . 7}$ & 90.7 & $\mathbf{8 4 . 6}$ & $\mathbf{9 5 . 7}$ \\
\hline GS fused bands & 87.3 & 90.0 & $\mathbf{8 3 . 5}$ & $\mathbf{9 3 . 2}$ \\
GS + Texture PAN & $\mathbf{8 7 . 8}$ & $\mathbf{9 0 . 3}$ & $\mathbf{8 3 . 5}$ & $\mathbf{9 3 . 2}$ \\
GS + Indices PAN & 85.7 & 90.2 & $\mathbf{8 3 . 5}$ & $\mathbf{9 3 . 2}$ \\
\hline
\end{tabular}

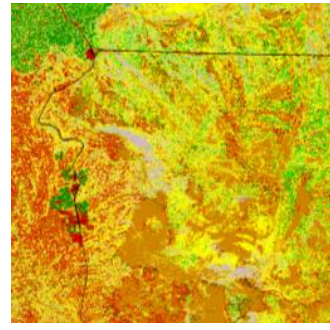

(a)

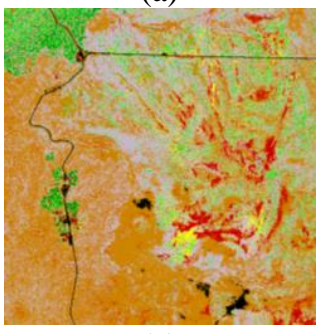

(c)

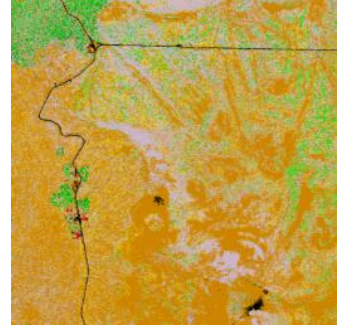

(b)

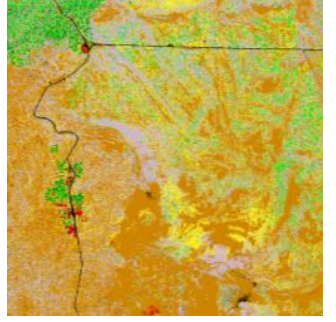

(d)
Figure 5. Thematic maps for each classifier: (a) MhD, (b) ML, (c) SAM y (d) SVM. 
Figure 6 shows results of the ML and SVM classifiers, for a zoomed area considering the best original and fused band combinations. It can be appreciated the spatial improvement when using the fused information and the superior performance of SVM when compared to a real photograph of the area.

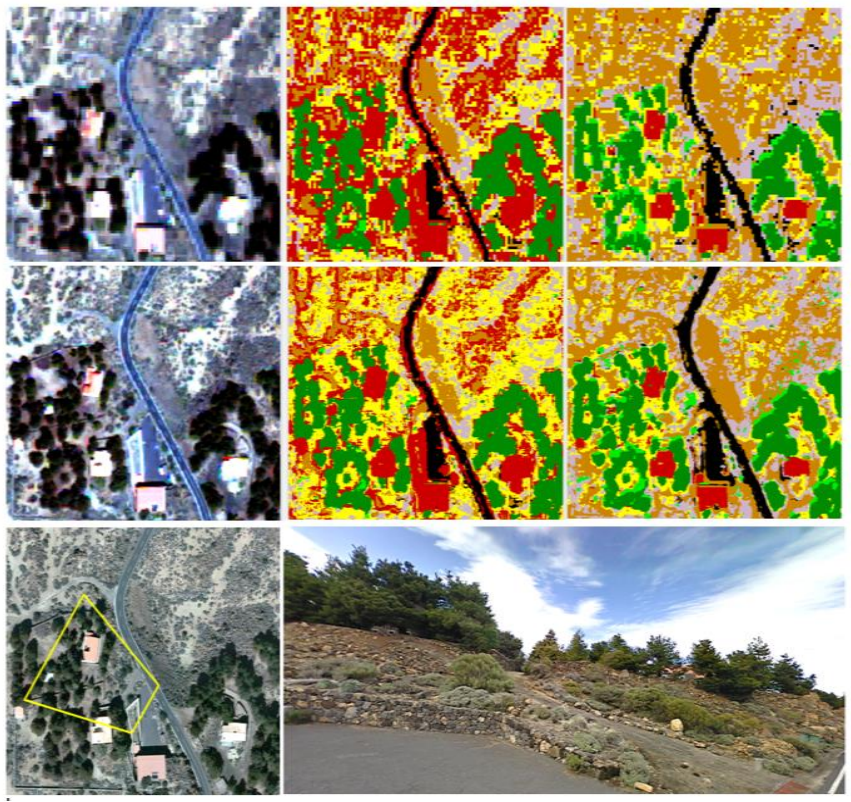

Figure 6. ML ( $2^{\text {nd }}$ column $)$ and SVM ( $3^{\text {rd }}$ column $)$ maps for the original ( $1^{\text {st }}$ row) and pansharpened ( $2^{\text {nd }}$ row) bands. Last row includes a $12 \mathrm{~cm} /$ pixel orthophoto (Feb. 27, 2011) and a real Google streetview photograph (Jan. 2009).

\section{CONCLUSIONS}

The mapping of vegetation covers in semi-arid natural areas is a difficult task as, typically, such places are sparsely vegetated and mainly composed by small shrubs of different species. In this complex scenario, this paper presents some ideas and intermediate results for different approaches to the data fusion problem of multisensory data applied to protected vegetated areas in Canarian Islands. Either of the data fusion framework has the selection of input datasets as one of the most delicate steps in the procedure, in particular due to the temporal gap among datasets. Fusion procedures may present different levels of complexity according to whether the fusion is performed at decision, feature or pixel levels but show a huge potential, especially if applied to classification of vegetated areas in which spectral similarities of different species may make the use of single source data unsuccessful. Currently the authors are in the step of completing the implementation of the fusion chains and final results will be presented at the conference.

\section{ACKNOWLEDGEMENTS}

This work has been supported by the ARTEMISAT (CGL2013-46674-R) project, funded by the Spanish Ministerio de Economía y Competitividad and the Surrey Space Centre, University of Surrey The authors would like to express their gratitude to DLR for providing TerraSAR-X data within the project LAN2914.

\section{REFERENCES}

[1] L. Alparone, S.Baronti, A.Garzelli, F.Nencini, "Landsat ETM+ and SAR Image Fusion Based on Generalized Intensity Modulation", IEEE Transactions on Geoscience and Remote Sensing, vol.42, n.12, pp. 2832-2839, 2004.

[2] Vermote E., D. Tanré, J. L. Deuzé, M. Herman, J. J. Morcrette, and S. Y. Kotchenova, Second Simulation of a Satellite Signal in the Solar Spectrum - Vector (6SV), 6S User Guide Version 3, 2006.

[3] S. Hantson, and E. Chuvieco, "Evaluation of different topographic correction methods for Landsat imagery," International Journal of Applied Earth Observation and Geoinformation, vol. 13, pp. 691-700, 2011.

[4] G. Vivone, L. Alparone, J. Chanussot, M. Dalla Mura, A. Garzelli, G. Licciardi, Restaino and L. Wald, "A Critical Comparison Among Pansharpening Algorithms," IEEE Transactions on Geosciences and Remote Sensing, v. 53, n.5, pp.2565-2586, 2015.

[5] J. Marcello, A. Medina, and F. Eugenio, "Evaluation of spatial and spectral efectiveness of pixel-level fusion techniques," IEEE Geoscience and Remote Sensing Letters, vol.10, pp. 432-436, 2013.

[6] Tso, B. and PM. Mather, Classification Methods for Remotely Sensed Data, Taylor and Francis Inc., New York, 2009.

[7] G. Mountrakis, J. Im and C. Ogole, "Support vector machines in remote sensing: A review," ISPRS Journal of Photogrammetry and Remote Sensing, vol. 66, pp. 247-259, 2011.

[8] X. Yang, "Parameterizing Support Vector Machines for Land Cover Classification," Photogrammetric Engineering and Remote Sensing, vol. 77 (1), pp. 27-37, 2011. 